Implantologie

\title{
Für den atrophierten Kieferkamm
}

Speziell für den schräg atrophierten Kieferkamm hat DENTSPLY Implants ein Implantat entwickelt: das OsseoSpeed Profile EV. EV steht für ,Evolution` und damit für das Konzept der kontinuierlichen Weiterentwicklung des ASTRA TECH ImplantSystem. Mit seinem einzigartigen, abgeschrägten Implantatschulter-Design folgt das Implantat der natürlichen Form des Knochens und ermöglicht mit einem $360^{\circ}$-Knochenerhalt rund um das Implantat eine verbesserte Weichgewebsästhetik. In vielen Fällen lässt sich so eine Augmentation vermeiden. Die Prothetik und das Handling der ersten Profile-Serie aus dem Jahr 2011 wurden bei dem neuen Implantat komplett überarbeitet. Hervorzuheben ist eine sehr einfache Abdrucknahme durch die ,One-positiononly'-Platzierung. Der selbstpositionierende Abdruckpfosten vereinfacht die Abdrucknahme und hilft, Fehler zu vermeiden: Beim Festziehen der Schraube dreht sich der Pfosten in die richtige Po-

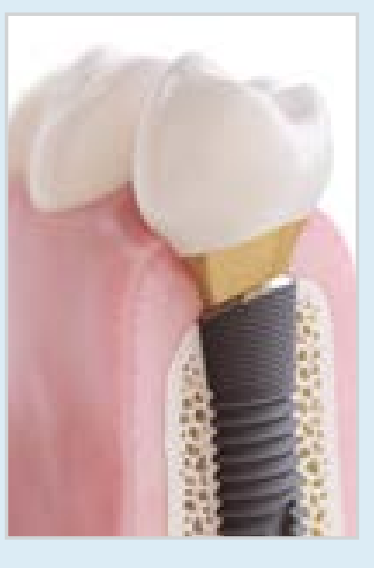
sition. Nur wenn er richtig sitzt, greift er in das Implantat ein. DENTSPLY Implants wird mit dem OsseoSpeed Profile EV-Implantat erneut seinem Anspruch gerecht, für jede Indikation das ideale Implantat anbieten zu können.

Nach einer Pressemitteilung der

DENTSPLY IH GmbH, Mannheim 